\title{
Cortejo y Apareamiento del Puma yagouaroundi en el Zoológico Rosy Walther, Honduras
}

\section{Francisco Aceituno ${ }^{1}$}

Resumen. En esta nota se describe el cortejo y apareamiento observado entre una pareja de yaguarundí en cautiverio. Adicionalmente se describe la dieta, las dimensiones del encierro y el comportamiento social de los individuos. Las observaciones se realizaron en el zoológico Rosy Walther en Tegucigalpa, Honduras. El comportamiento asociado a la cópula fue el típico de los pequeños felinos, la etapa precopulatoria fue caracterizada por la persecución y acercamiento del macho. Cuando la hembra estaba sexualmente receptiva permitió que el macho se acercara a ella, él le mordió la nuca y ella aceptó la cópula y se agachó permaneciendo en el suelo, finalmente la hembra fue montada por el macho. Desde el año 2012, solamente se ha registrado el nacimiento de una cría, sin embargo el cachorro fue encontrado muerto dentro del recinto.

Palabras clave: Cautiverio, copula, recinto, yaguarundí.

\section{Courtship and Mating of the Puma yagouaroundi in the Rosy Walther Zoo, Honduras}

\begin{abstract}
This note describes the courtship and mating exhibited by a pair of jaguarundi in captivity. Additionally, the diet, enclosure size and social behavior of the species are described. The observations were made at the Rosy Walther Zoo Tegucigalpa, Honduras. The behavior associated with copulation was typical of small felids; the precopulatory phase was characterized by chase and male approach. When the female was susceptible to sexual activity she allowed the male close to her, he grasped her by the nape, she accepted copulation and crouched, remaining flat on the ground, and finally she was mounted by the male. Since 2012, there has been recorded one kitten born; however the cub body was found dead inside the enclosure.
\end{abstract}

Key words: Captivity, copulation, enclosure, jaguarundi.

\section{Introducción}

El yaguarundí (Puma yagouaroundi) es un felino de tamaño pequeño a mediano con un peso promedio de $5 \mathrm{~kg}$, tiene cuerpo alargado, orejas redondeadas, patas cortas, cabeza pequeña y presenta dos fases de coloración; la fase café rojizo y la fase grisáceo (Oliveira 1998, Caso et al.2015). La especie presenta ciclos estrales durante todo el año, en intervalos de 53 a 56 días, el periodo de gestación es de unos 75 días; el tamaño de la camada fluctúa de una a cuatro crías y alcanzan la madurez sexual entre los dos y tres años de edad (Nowell y Jackson 1996). En relación a otros felinos neotropicales son pocos los trabajos de investigación sobre esta especie, por tanto aspectos sobre su ecología son poco conocidos y la mayoría de los datos que se ha logrado documentar, son debido a las facilidades que permiten los estudios en condiciones de cautiverio (Giordano 2015, Shostell y Ruiz-García 2013, Mellen 1993).

Mellen (1991) observó los factores ambientales, sociales y genéticos que intervienen en la reproducción de los felinos en cautiverio. Los resultados presentados en este estudio indican que las condiciones de los encierros en relación a calidad y complejidad, la disposición de los grupos de animales y mayor tiempo de interacción entre el cuidador y el animal pueden favorecer las condiciones para su reproducción. Este mismo autor sugiere que el aumento en la frecuencia de conductas sociales como marcas de orina, marcas con garras, frotamiento de cabeza y mejillas, además de la observación de comportamientos de persecución y monta pueden servir como parámetros para indicar el inicio del estro y la actividad reproductiva en felinos ex situ (Mellen 1993).

\footnotetext{
${ }^{1}$ Sub Unidad de Biología y Veterinaria, Zoologico Rosy Walther, Secretaria de Energía, Recursos Naturales, Ambiente y Minas. Tegucigalpa, Honduras. E-mail: aceitunof@yahoo.com
} 
Los zoológicos han empezado a establecer programas de reproducción exitosos. Sin embargo es necesario promover esfuerzos para mejorar la representación de especies de pequeños felinos en estas colecciones, además es crítica la generación de información de la biología de estas especies en condiciones de cautividad (Nowell y Jackson 1996). Esta nota tiene como objetivo describir el cortejo y apareamiento del Puma yagouaroundi, en el Zoologico Rosy Walther, de manera que puedan servir como referencia para mejorar el bienestar de la especie en condiciones ex situ.

Individuos. El zoológico Rosy Walther alberga dos individuos de yaguarundí; ambos nacieron en vida silvestre posteriormente capturados y donados a este centro. El macho de coloración café grisáceo ingresó en el año de 2011 y la hembra de coloración rojiza ingresó en el 2012. Los datos sobre los hábitos y comportamientos de ambos individuos fueron recolectados mediante observaciones ad libitum, durante diciembre de 2013 y enero de 2014.

Recinto y Dieta de los Individuos. Los yaguarundí del zoológico Rosy Walther se encuentran alojados en un recinto que mide $7 \mathrm{~m}$ de largo $\times 6.20 \mathrm{~m}$ de ancho con una altura de $3 \mathrm{~m}$, tiene piso de tierra $\mathrm{y}$ paredes de malla metálica que también cubren el techo y las puertas, lo que facilita la observación de los ejemplares. El encierro está divido en dos secciones que están comunicados por una puerta, esto permite separar los individuos si es necesario. Como parte de la ambientación del recinto se han colocado pequeños troncos de madera que sirven para descanso, además tienen un comedero y un bebedero.

El encierro cuenta con dos casas dormidero de madera que miden $95 \mathrm{~cm}$ de ancho, $1.4 \mathrm{~m}$ largo y 70 $\mathrm{cm}$ de altura; se encuentra sobre una plataforma a una altura de $1.2 \mathrm{~m}$ sobre el suelo. En las mismas se han colocado troncos que permiten a los animales trepar a las casas. La limpieza es todos los días a las 8:00h, posteriormente son exhibidos al público a partir de las 9:00h hasta las 15:00h.

La dieta de los individuos consiste de una ración de $0.45 \mathrm{~kg}$ de carne vacuna al día para cada individuo. La carne se corta un día antes y se mantiene en refrigeración para dársela a los ejemplares a las $1400 \mathrm{~h}$. La comida permanece en el comedero hasta el día siguiente por la mañana cuando los restos no consumidos son removidos por los cuidadores. Como parte de las actividades de enriquecimiento ambiental alimenticio, se les ofrece también tilapia roja (Oreochromis mossambicus) y carne de pollo, alimentos que son bien aceptados por los yaguarundí.

Comportamiento Social. En relación a las interacciones sociales, la mayor parte del día los individuos se dedican a descansar y empiezan a estar activos alrededor de las 10:00h; el macho presenta mayor actividad que la hembra. Los comportamientos de tipo social en pequeños felinos en condiciones de cautiverio solo representan del 1 al $2 \%$ de las actividades realizadas por los animales e incrementan su actividad en presencia de estímulos de enriquecimiento ambiental (Mellen 1993, Resende et al. 2009).

A ambos individuos les gusta arañar los palos que utilizan para trepar a las casas, asimismo el macho realiza marcas de orina en pequeños montículos de tierra que ha acumulado cerca de la base de las casas de madera, a diferencia de la hembra que durante el tiempo de observación solamente exhibió este comportamiento de marcaje por orina en una ocasión. Estas observaciones coinciden con los hallazgos de Hulley (1976), Bennett y Mellen (1983) y Mellen (1993) que reportan que los yaguarundí marcan y arañan los objetos que se encuentran en los recintos para marcar territorio, además este comportamiento es común en época de apareamiento y más frecuente en machos.

\section{Descripción del Comportamiento Reproductivo}

En el comportamiento de cortejo y cópula de los individuos se diferenciaron tres etapas; inicialmente el cortejo comenzó con un aumento en la frecuencia de actividad del macho buscando contacto con la hembra, la persiguió y esta respondió con un comportamiento de agresión. En segunda instancia la hembra se mostró más receptiva, el macho la olió hasta que esta permitió que se le acercara, seguidamente el macho la mordisqueó en la nuca y la hembra flexionó los miembros y se echó en el suelo. Finalmente el macho sostuvo a la hembra y se apoyó en las patas traseras de esta, inmediatamente la montó vigorosamente por la parte posterior; la hembra es más pequeña lo que permitió al macho montarla con más facilidad. Las montas duraron en promedio 20 segundos (Figura 1). 

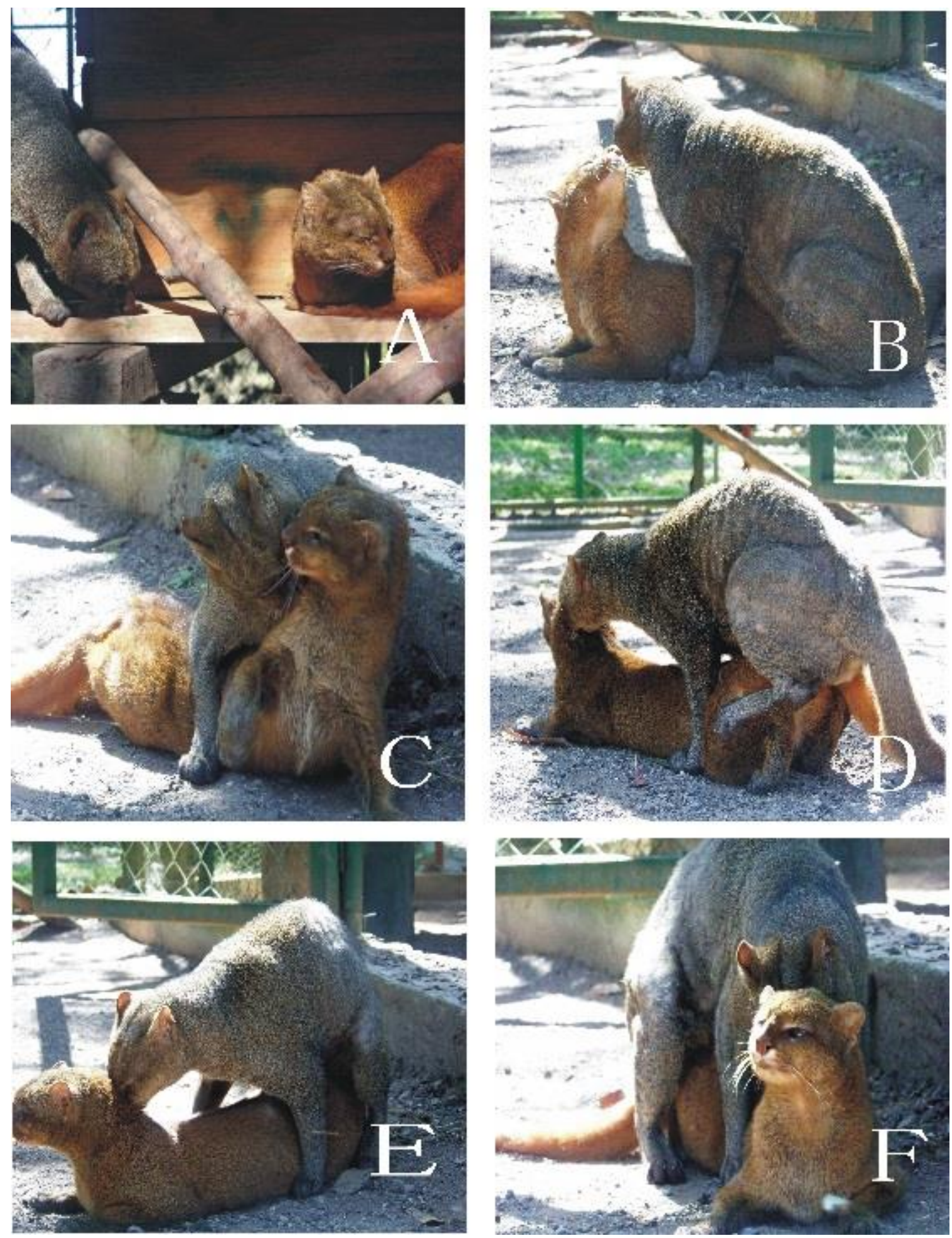

Figura. 1. Secuencia fotográfica del cortejo y apareamiento del yaguarundí en el zoológico Rosy Walther, Tegucigalpa, Honduras. A, B y C comportamiento del cortejo precopulatorio, el macho busca la hembra hasta que cede a su presencia. D, E y F el macho monta la hembra y se consuma la cópula. 
.Desde que los individuos se encuentran en el zoológico, solamente se ha registrado el parto de una cría que fue encontrada muerta en el interior del recinto. Al espécimen se le registraron las medidas externas básicas (longitud total $138 \mathrm{~mm}$, longitud pata anterior izquierda $16 \mathrm{~mm}$, longitud de la pata posterior izquierda $27 \mathrm{~mm}$, longitud de la oreja $8 \mathrm{~mm}$ y el peso $0.11 \mathrm{~kg}$ ) posteriormente fue preparada en formalina al $90 \%$ y donada a la colección de mamíferos del Museo de Historia Natural de la Universidad Nacional Autónoma de Honduras. No fue posible determinar la causa de muerte, sin embargo, la cría presentaba mutilada la cola y cuando se intentó removerla del recinto la hembra se mostró agresiva. En condiciones de cautiverio las hembras carnívoras presentan dificultades para cuidar los cachorros, en este sentido las principales causas de mortalidad de pequeños felinos en estas condiciones están relacionadas con agresiones por rechazo o negligencia por parte de la madre (Von Schmalz-Peixoto 2003).

\section{Discusión}

En la actualidad, la mayoría de iniciativas y esfuerzos de investigación están orientados a los grandes felinos, en contraste con los felinos pequeños sobre los que se requieren investigaciones acerca de su estado de conservación y biología (Nowell y Jackson 1996, Giordano 2015). Los programas de propagación en cautiverio representan una importante alternativa en las iniciativas de conservación internacional, ya que en los zoológicos se albergan especies que están amenazadas, además estas instituciones generan información científica (Swanson, et al. 2003). Un paso previo para el desarrollo de estos programas es realizar investigaciones que permitan conocer los aspectos básicos de la biología de la especies en cuestión. Los estudios sobre mamíferos en cautiverio son limitados, en consecuencia, los zoológicos y centros de rescate pueden ser actores relevantes en la generación de este tipo de información para disponer de datos y mejorar el manejo de estas especies.

\section{Literatura Citada}

Bennett, S.W. y J. D. Mellen. 1983. Social interactions and solitary behaviors in a pair of captive sand cats (Felis margarita). Zoo Biology 2:39-46.

Caso, A.,T. de Oliveira y S.V. Carvajal. 2015. Herpailurus yagouaroundi. The IUCN Red List of Threatened Species 2015: Downloaded on 27 November 2015.

Giordano, A.J. 2015.Ecology and status of the jaguarundi (Puma yagouaroundi): a synthesis of existing knowledge. Mammal Review 46:30-43.

Hulley, J.T. 1976. Maintenance and breeding of captive jaguarundis (Felis yagouaroundi) at Chester Zoo and Toronto. International Zoo Yearbook 16:120-122.

Mellen, J. 1991. Factors influencing reproductive success in small captive exotic felids (Felis spp.): A multiple regression analysis. Zoo Biology 10:95-110.

Mellen J. 1993. A comparative analysis of scentmarking, social and reproductive behavior in 20 species of small cats (Felis). American Zoologist 33:151-166.

Nowell, K. y P. Jackson. 1996. Wild cats: status survey and conservation action plan. Gland, Switzerland: IUCN/SSC Cat Specialist Group. 382 p.

Oliveira, T. 1998. Herpailurus yagouaroundi. Mammalian species 578:1-6.

Resende, L.S., G.L. Remy., V.A.Ramos y A. Andriolo. 2009. The influence of feeding enrichment on the behavior of small felids (Carnivora: Felidae) in captivity. Zoología 26:601-605.

Shostell, J.M. y M. Ruiz-Garcia. 2013. An introduction to neotropical carnivores. In: Molecular population genetics, evolutionary biology and biological conservation of the Neotropical carnivores. p 1-36.

Swanson, W.F., W.E. Johnson., R.C. Cambre., S.B. Citino., K.B. Quigley., D.M Brousset., R.M. Morais., N. Moreira., S.J. O'Brien y D.E. Wildt. 2003. Reproductive status of endemic felid species in Latin American zoos and implications for ex situ conservation. Zoo Biology 22:421-441.

Von Schmalz-Peixoto, K. 2003. Factors affecting breeding in captive Carnivora. Ph. D. Thesis, University of Oxford, United Kingdom.

Recibido para publicación el 22 de octubre del 2015. Aceptado para publicación el 11 de enero del 2016. 